\title{
Wnt-5a Mimic Hexapeptide Foxy-5
}

National Cancer Institute

\section{Source}

National Cancer Institute. Wht-5a Mimic Hexapeptide Foxy-5. NCI Thesaurus. Code C113435.

A formylated, six amino acid, Wnt5a-derived peptide and wnt-5a mimetic with potential anti-metastatic activity. Upon intravenous administration, Wnt-5a mimic hexapeptide foxy- -5 binds to and activates the wnt-5a receptors, Frizzled- 2 and -5 , which activates wnt-5a-mediated signaling. Increased wnt-5a signaling may inhibit endothelial tumor cell migration and invasion. This may decrease metastasis of susceptible tumor cells. However, foxy-5 does not affect tumor cell proliferation or apoptosis. Foxy-5 lacks a heparan sulfate-binding domain and contains a formyl group on its $\mathrm{NH}$-terminal methionine residue which decreases in vivo degradation. Decreased expression of wnt-5a protein is associated with increased motility of certain tumor cell types. 